\section{Escolha de métodos contraceptivos entre usuárias de um serviço público de saúde}

\author{
Choice of contraceptive methods by women \\ attending a public health clinic
}

Maria José Duarte Osis 1 Graciana Alves Duarte 1 Evely Rodrigues Crespo 1 Ximena Espejo 2 Karla Simônia de Pádua 1

\footnotetext{
1 Centro de Pesquisas Materno-Infantis de Campinas, Campinas, Brasil. 2 Centro de Assistência Integral à Saúde da Mulher, Universidade Estadual de Campinas, Campinas, Brasil.

Correspondência M. J. D. Osis Centro de Pesquisas Materno-Infantis de Campinas. C. P. 6181, Campinas, $S P$ 13084-971, Brasil. mjosis@cemicamp.org.br
}

\section{Abstract}

This paper presents the perceptions of 250 women who requested contraceptive methods (CM) at a public health clinic in relation to their freedom of choice and the role of an educational activity and consultation in this degree of freedom. Satisfaction with the chosen method and continuation after 6 months were also studied. Nearly all the women (99.6\%) had already chosen a CM when they came to the clinic, and 90.0\% left the clinic with the method; $81.9 \%$ reported that they felt very free to choose the method, and $60.0 \%$ felt that the educational activity and consultation had improved their degree of freedom. Six months later, $87.3 \%$ were using the same CM they had chosen. The proportion of women who changed the method and the proportion of those who felt they had not received sufficient information about the CM when they initiated use were significantly higher among women who reported any dissatisfaction with that method. The educational activity and consultation legitimated the women's choices (which they had already made before attending the health clinic) on the basis of such supplementary sources of information. This fact appears to have contributed to the women's feeling that they had sufficient freedom of choice.

Contraception; Family Planning; Health Services

\section{Introdução}

No contexto dos direitos reprodutivos, a liberdade de escolha é fundamental na área da regulação da fecundidade. Para optar por um método contraceptivo de forma livre e informada, as mulheres precisam conhecer e ter acesso a todos os métodos anticoncepcionais (MAC) cientificamente aprovados e disponíveis, escolhendo aquele que seja mais adequado às suas características e às suas condições de vida em cada momento. Dentro dessa proposta, o planejamento familiar tem como objetivo garantir às mulheres e aos homens um direito básico de cidadania: o direito de ter ou não filhos/as 1,2 . Ao final da década de 1980, foi publicado um marco conceitual, até hoje utilizado como referência para qualidade de atenção em planejamento familiar, que inclui seis elementos fundamentais: escolha livre de métodos, informação para usuárias, competência técnica, relação usuária-serviço; acompanhamento de usuárias e integração do planejamento familiar ao atendimento em saúde reprodutiva 3 .

O processo de escolha informada na regulação da fecundidade baseia-se nos princípios de proporcionar bem-estar às pessoas, quanto à sua autonomia, expectativas, necessidades e poder de decisão, enfocando especialmente os direitos sexuais e reprodutivos, na qualidade de direitos humanos individuais. Embasados em premissas como essas, muito se tem reivin- 
dicado que os programas voltados à regulação da fecundidade, ao planejamento familiar, incluam sempre um componente educativo, muitas vezes chamado de ação educativa, visando a dar subsídios às pessoas para escolherem de forma livre e informada qual contraceptivo usar 4 .

Para que a opção por um método seja livre e informada, além da variedade, também é necessário ter um número suficiente a ser oferecido, levando-se em conta as características intrínsecas de cada pessoa e a demanda local. Além disso, para que as pessoas, de fato, possam escolher livremente, é relevante também a informação científica que lhes é dada acerca dos MAC disponíveis. Neste ponto, incluem-se as contra-indicações gerais e as situações próprias de cada indivíduo que poderiam levar a considerar determinados MAC como mais ou menos adequados para essa pessoa, e, finalmente, o tipo de suporte que os serviços podem oferecer às pessoas que escolhem um método específico 3 .

A literatura também tem mostrado que uma boa interação provedor-cliente não só contribui para a liberdade de escolha, como também em passar informações precisas em um processo que proporcione um clima de confiança, permitindo troca de informações, em duas vias. Para isso, alguns princípios deveriam ser respeitados: tratar bem o cliente, oferecer o MAC que ele deseja, dar uma atenção individualizada, visar à interação dinâmica, evitar sobrecarga de informação e utilizar auxílios visuais para a memorização ${ }^{5}$. Além de adotar este papel mais ativo na oferta de ações educativas, o provedor não deve esquecer as circunstâncias de vida, seja pessoal ou familiar, que envolvem as mulheres que buscam um método para regular sua fecundidade, nem do seu estado psicológico e físico ${ }^{6}$. Assim, acredita-se ser possível obter uma decisão mais consciente, em que vantagens e desvantagens de cada MAC oferecido são pesadas na hora de decidir. A continuação do uso de um método escolhido estará positivamente associada a ter recebido uma orientação de boa qualidade, entrelaçando-se aí a disponibilidade de contraceptivos tradicionais e novos 7 .

No Brasil, desde a implantação do Programa de Assistência Integral à Saúde da Mulher (PAISM), a partir de 1984, tem havido a preocupação de melhorar a qualidade da atenção em planejamento familiar. Isto levou vários serviços públicos de saúde a oferecerem, pelo menos uma ação educativa às mulheres que os procuram para solicitar MAC. Em 1996, um projeto de lei aprovado pelo Congresso Nacional (Lei 9.263), sancionado pela Presidência da República, regulamentou o planejamento familiar. Formalmente, esta lei democratiza o acesso aos meios contraceptivos nos serviços públicos de saúde, assim como regulamenta essa prática na rede privada, sob controle do SUS. Em 2001, a última Norma Operacional de Assistência à Saúde (NOAS-2001) do Ministério da Saúde colocou a assistência em planejamento familiar entre as ações mínimas que devem ser implementadas em todos os municípios 1,2 .

Apesar dessa ênfase programática, concretizar tais ações é um desafio. Sabe-se que na área de planejamento familiar existem muitas dificuldades para ofertar, em diferentes localidades no Brasil, uma atenção com qualidade. Existem vários pontos de estrangulamento, como por exemplo, deficiências na infra-estrutura para a assistência (área física, recursos humanos, equipamentos, instrumental, material de consumo e educativo e formulários de registro); logística de MAC (métodos adequados à demanda e apropriadamente distribuídos); divulgação do serviço de planejamento familiar, não somente à demanda espontânea; capacitação do pessoal de saúde; comunicação entre as esferas municipal e federal e dificuldades geográficas 1,2,8,9. Quanto ao componente educativo em planejamento familiar no Brasil, não se encontram descrições na literatura, sabe-se que vários serviços preocupam-se em orientar as pessoas que buscam MAC. O Ambulatório de Planejamento Familiar do Centro de Assistência Integral à Saúde da Mulher (CAISM), da Universidade Estadual de Campinas (UNICAMP), é um desses serviços.

O referido ambulatório é um centro de referência em anticoncepção e desenvolve atividades de assistência, docência e pesquisa. Conta com uma equipe multidisciplinar constituída de médicos, enfermeiras, assistente social e psicóloga. Desenvolve ações educativas com as mulheres que chegam em busca de métodos anticoncepcionais e permite aos homens também a possibilidade de participarem dessas atividades. Uma freqüente discussão entre os profissionais que atuam nesse ambulatório refere-se à qual é a contribuição das ações educativas para a escolha livre e informada, levando em conta as expectativas das mulheres em relação aos MAC, quando chegam ao serviço. Este artigo tem como objetivo descrever a percepção de mulheres que solicitaram um MAC 
nesse Ambulatório, quanto à sua liberdade de escolha, à contribuição da ação educativa e da consulta para essa liberdade e à continuidade de uso do MAC escolhido, depois de seis meses, e à satisfação ou não com o mesmo.

\section{Material e métodos}

Foi desenvolvido um estudo descritivo, longitudinal. O tamanho amostral foi calculado em 210 sujeitos, com base em um estudo realizado por Walsh et al. 10 , considerando-se que a proporção estimada de mulheres satisfeitas com a informação recebida do profissional de saúde sobre o método contraceptivo escolhido foi de $73,0 \%$, uma diferença (precisão) desejada entre a proporção amostral e populacional (d) de 6,0\% e erro tipo I de 5,0\%. Prevendo-se uma possível perda de seguimento de $16,0 \%$, decidiu-se a inclusão de 250 sujeitos no estudo. A razão para utilizarem-se resultados de um estudo realizado fora do Brasil para calcular o tamanho da amostra foi o não-conhecimento de estudos nacionais que contivessem as informações necessárias.

Entrevistaram-se 250 mulheres que chegaram ao Ambulatório de Planejamento Familiar do CAISM em busca de um MAC. Paralelamente, foi feita observação de cinco ações educativas, escolhidas ao acaso. O protocolo da pesquisa foi avaliado e aprovado pela Comissão de Pesquisa do Departamento de Tocoginecologia e pelo Comitê de Ética em Pesquisa, ambos da Faculdade de Ciências Médicas da UNICAMP. A participação das mulheres no estudo foi voluntária e desvinculada de seu atendimento no ambulatório, mediante assinatura de Termo de Consentimento Livre e Esclarecido.

A coleta de dados ocorreu entre 26 de novembro de 2001 e 15 de dezembro de 2002. Para seleção e entrevista das mulheres, foram treinadas duas entrevistadoras, desvinculadas do ambulatório; as ações educativas foram observadas por duas das pesquisadoras responsáveis pelo estudo.

A seleção das mulheres deu-se dentro da rotina de atendimento do serviço em questão. Diariamente, as entrevistadoras acompanharam o atendimento no ambulatório e identificaram as mulheres que estavam chegando ali pela primeira vez e que eram encaminhadas para uma ação educativa. Nessa ação, são abordados todos os métodos disponíveis no serviço, suas características, indicações e contra-indicações. Neste momento é dada a oportunidade para perguntas e discussão entre as(os) participantes-provedor. A seguir, elas passam por uma consulta individual quando informam qual método desejam usar, e também se observa o grau de segurança que têm ao fazer essa opção. É feita uma avaliação quanto a se apresentam ou não contra-indicações ao uso do MAC escolhido. Em caso positivo, discutem-se as opções. Diante de alguma dúvida ou indecisão, reforçam-se as informações necessárias e/ ou convida-se a mulher a refletir um pouco mais sobre o assunto e discuti-lo com o parceiro, se possível. Abre-se a possibilidade de que ela retorne em outro dia, se julgar que precisa de mais tempo para a decisão.

No mesmo dia em que era selecionada e aceitava participar do estudo, cada mulher foi entrevistada em dois momentos, utilizando-se um questionário estruturado e pré-testado, antes de assistir à ação educativa, quando foram aplicadas as duas primeiras seções desse questionário, e depois da consulta, quando era aplicada a seção 3 desse mesmo instrumento. Foi providenciado um local apropriado para que as entrevistas fossem conduzidas em privacidade no próprio ambulatório de reprodução humana. Ao encerrar a primeira entrevista, era agendado um retorno da mulher ao serviço para uma nova entrevista seis meses depois. Nessa ocasião, utilizou-se um outro questionário estruturado e pré-testado.

Os questionários preenchidos foram revisados e as respostas pré-codificadas foram digitadas diretamente valendo-se deles. Para as respostas às perguntas abertas, primeiramente foi preparado um Manual de Codificação, com base em uma amostra das respostas a cada pergunta. Em seguida, foi realizada a codificação, também por duas pessoas diferentes, que discutiam as discordâncias na atribuição dos códigos, de modo a chegarem a um consenso. Depois disto, os códigos eram digitados. O programa de entrada dos dados permitia que a segunda digitação fosse interativa com a primeira, checando-se a consistência entre as duas e corrigindo-se imediatamente os erros. Após a entrada completa dos dados, foi realizada uma análise de consistência lógica dos mesmos. Para todos os procedimentos de digitação e checagem foi utilizado o módulo de entrada de dados do Statistical Package for Social Science (SPSS).

Para a análise dos dados foi utilizado o programa estatístico SPSS for Windows 8.0. Neste trabalho apresenta-se, de início, uma análise descritiva univariada com as variáveis originais. Em seguida, foi executada análise bivariada. O teste qui-quadrado 11 foi utilizado para testar a associação entre as variáveis. 


\section{Caracterização da amostra}

$\mathrm{Na}$ amostra estudada, mais da metade das mulheres tinha idade de até 29 anos $(56,4 \%)$, possuía no máximo oito anos de escolaridade $(60,4 \%)$ e não realizava trabalho remunerado $(59,2 \%)$. A grande maioria das participantes era casada ou vivia em união $(87,6 \%)$ e declarou ter alguma religião $(92,4 \%)$. Pouco mais de um terço $(37,6 \%)$ das mulheres referiu renda familiar de até três salários mínimos e um quarto declarou renda acima de cinco salários mínimos. Quando perguntadas sobre a sua cor de pele, $55 \%$ das entrevistadas declararam-se negras ou morenas.

Quase todas as mulheres já haviam engravidado alguma vez, no entanto, pouco mais de um terço $(36,0 \%)$ referiu três ou mais gestações; pouco mais de um quarto declarou três ou mais partos $(27,9 \%)$, três ou mais nascidos vivos $(26,7 \%)$ e três ou mais filhos vivos $(25,8 \%)$. Três quartos $(76,2 \%)$ das entrevistadas declararam nunca ter tido abortos, e quase todas $(96,7 \%)$ disseram não ter havido nascidos mortos.

\section{Resultados}

Entre os MAC que as mulheres pretendiam usar ao chegarem ao ambulatório, o preferido foi o DIU (59,0\%), seguido da laqueadura $(24,0 \%) \mathrm{e}$ injeção (13,0\%). O método menos pretendido foi a pílula $(3,2 \%)$. Apenas uma mulher $(0,4 \%)$ chegou ao ambulatório sem saber qual MAC queria usar. As principais razões dadas pelas entrevistadas, quando chegavam ao ambulatório, para querer usar determinado método, referiam-se a ser um "método mais seguro/confia mais" (28,5\%) e que "outros MAC faziam mal/davam efeitos indesejados” (25,3\%). Quase metade das entrevistadas ouviu falar pela primeira vez do MAC desejado por meio de familiares; um pouco mais de um quarto referiu "médico/outros profissionais de saúde”; e um pouco menos de um quarto mencionou "outra mulher/pessoa/amigas/vizinhas". A idéia de usar o MAC foi das próprias mulheres ou de ninguém $(39,0 \%)$. Para cerca de um quarto delas, a idéia foi dada por um médico/outros profissionais de saúde (Tabela 1 ).

Após ter assistido à ação educativa e passado pela consulta, a grande maioria $(90,0 \%)$ escolheu usar o mesmo método pretendido inicialmente. Dentre as mulheres que escolheram outro MAC, as principais razões dadas para isso foram: não cumprir requisitos legais, no caso da esterilização $(38,0 \%)$, ter contra-indicação para uso do MAC $(27,0 \%)$, ter recebido orienta-
Tabela 1

Distribuição percentual dos MAC (métodos anticoncepcionais) que as mulheres pretendiam usar ao chegarem ao ambulatório, quem Ihes falou dele pela primeira vez e quem deu a idéia de usarem.

\begin{tabular}{|c|c|}
\hline Categorias & $\%$ \\
\hline \multicolumn{2}{|l|}{ MAC pretendido } \\
\hline DIU & 59,4 \\
\hline Laqueadura & 24,5 \\
\hline Injeção & 12,9 \\
\hline Pílula & 3,2 \\
\hline \multicolumn{2}{|l|}{ Razões para pensar usar um determinado MAC } \\
\hline Mais seguro/confia mais & 28,5 \\
\hline Outros MAC faziam mal/davam efeitos indesejados & 25,3 \\
\hline Mais prático/não precisa se preocupar & 19,7 \\
\hline Número de filhos ideal/não quer mais filhos & 17,3 \\
\hline Não faz mal à saúde/sem efeitos & 10,8 \\
\hline Problemas de saúde & 8,4 \\
\hline Amiga/parente indicou & 5,6 \\
\hline Médico indicou & 5,2 \\
\hline Pensa em fazer laqueadura & 1,6 \\
\hline Não gostava/não queria usar outro & 0,4 \\
\hline Outras & 2,8 \\
\hline \multicolumn{2}{|l|}{ De quem ouviu falar do MAC pela primeira vez } \\
\hline Mãe/irmã/cunhada/outros parentes & 45,0 \\
\hline Médico/outros profissionais da saúde & 25,3 \\
\hline Através de outra mulher/pessoa/amigas/vizinhas & 22,4 \\
\hline Livro/revista/TV/rádio/palestras/cursos & 6,8 \\
\hline Não sabe/não lembra & 2,0 \\
\hline \multicolumn{2}{|l|}{ Quem deu idéia para usar MAC } \\
\hline Ela própria/ninguém & 39,0 \\
\hline Médico/outros profissionais de saúde & 28,9 \\
\hline Outros parentes & 12,1 \\
\hline Amiga/vizinha/pessoa conhecida & 7,6 \\
\hline Mãe/sogra & 7,2 \\
\hline Marido/companheiro & 6,4 \\
\hline Total* & 249 \\
\hline
\end{tabular}

* Não se aplica a uma mulher, porque respondeu que não sabia qual método queria usar.

ção na ação educativa para escolher MAC mais adequado (12,0\%) (Tabela 2).

Quando se observou a distribuição percentual das entrevistadas segundo quatro subcategorias relacionadas à sua autopercepção sobre a liberdade de escolha que tiveram, verificouse que quase todas as mulheres $(99,6 \%)$ disseram ter se sentido livres para escolher o método que aceitaram usar após a consulta. A grande maioria delas $(81,9 \%)$ referiu ter se sentido bastante livre para escolher. Para um pouco 
Tabela 2

Distribuição percentual das entrevistadas segundo MAC (métodos anticoncepcionais) que pretendiam usar quando chegaram ao ambulatório e o que foi escolhido após a ação educativa e consulta e razões para terem escolhido outro MAC.

\begin{tabular}{lr}
\hline Categorias & $\%$ \\
\hline MAC escolhido após a ação educativa e consulta & \\
O mesmo & 90,0 \\
Outro & 10,0 \\
& \\
Total* & 249 \\
Razões para terem escolhido outro MAC & \\
Não cumpriram requisitos legais (esterilização) & 38,0 \\
Contra-indicações para o uso do MAC & 27,0 \\
Orientadas a escolher MAC mais adequado & 12,0 \\
Consideraram outro MAC mais seguro & 4,0 \\
Outras & 23,0 \\
Total & 26 \\
\hline
\end{tabular}

* Uma mulher não havia escolhido MAC antes da ação educativa e da consulta. mais de dois terços (68,5\%), a ação educativa e a consulta tiveram alta influência na escolha do MAC, e três quintos $(60,0 \%)$ consideraram que essa ação aumentou a liberdade de escolha (Tabela 3).

Após seis meses de iniciado o uso do MAC escolhido, foi possível obter informações de 210 mulheres das 250 inicialmente admitidas ao estudo. Isto representou uma perda de seguimento de $15,2 \%$, dentro do que já se previra no cálculo do tamanho da amostra. A grande maioria das mulheres $(87,3 \%)$ entrevistadas seis meses depois de terem escolhido um MAC no Ambulatório continuava usando o mesmo método, e 85,0\% declararam estar muito satisfeitas/satisfeitas com ele. Em relação à informação recebida na ação educativa, $11,0 \%$ das mulheres disseram, após seis meses, que lhes havia faltado alguma informação sobre o MAC iniciado. A grande maioria das mulheres $(89,3 \%)$ afirmou que o uso do MAC lhes trouxera benefícios (Tabela 4 ).

Dentre as mulheres que se declararam muito satisfeitas/satisfeitas, $94,0 \%$ continuavam com o mesmo MAC após seis meses, comparadas a $50,0 \%$ daquelas que se disseram pouco satisfeitas/insatisfeitas, sendo a diferença estatisticamente significativa (Tabela 5). Dentre as mulheres que expressaram pouca satisfação/insatisfação, as principais razões para isto foram que o método faz mal à saúde/dá efeito colateral $(58,0 \%)$, seguida de que o MAC desregula a menstruação (31,0\%). Outras razões menos freqüentes foram: método pode falhar/mulher fica insegura; é difícil de usar/mulher precisa lembrar de usar/colocar/tomar o método e é desagradável usá-lo (dados não apresentados em tabela).

Das mulheres que se declararam muito satisfeitas/satisfeitas com o método escolhido, $8,2 \%$ consideravam ter faltado informação sobre o MAC na ação educativa e na consulta comparadas com $27,0 \%$ das entrevistadas que estavam pouco satisfeitas/insatisfeitas. Essa diferença também foi significativa. A proporção de mulheres que disseram estar muito satisfeitas/satisfeitas com o MAC e também achavam que este lhes trouxe benefícios, foi quase a totalidade $(97,2 \%)$. Dentre as que se sentiram pouco satisfeitas/insatisfeitas, um pouco mais de dois quintos $(42,0 \%)$ disseram que o método lhes trouxe benefícios (Tabela 5).

Em relação à ação educativa ministrada diariamente no Ambulatório de Planejamento Familiar, as pesquisadoras fizeram cinco observações em oportunidades distintas. Em todas as ocasiões a ação educativa foi dirigida por uma enfermeira capacitada. As ações observadas duraram de 40 a 54 minutos, e o número de participantes variou de cinco a $15 \mathrm{mu}$ lheres. Apenas em uma ocasião houve um homem participando da ação educativa, acompanhando a esposa.

A enfermeira-educadora sempre procurou estabelecer uma relação cordial com o grupo de mulheres que participava da ação. Ela iniciava a ação educativa identificando a demanda das mulheres quanto ao método que pretendiam usar e o que sabiam a respeito dele.

Em geral, o método utilizado pela enfermeira-educadora para ministrar as ações, era material ilustrativo (álbum seriado) para abordar cada MAC e o próprio método (DIU, diafragma etc.), que circulava entre as participantes para que pudessem observar e tocar. Em todas as ações observadas foi dada oportunidade para as mulheres fazerem perguntas, que foram respondidas adequadamente. As mulheres, em geral, utilizaram muito pouco a oportunidade para fazer perguntas. Nem sempre, porém, houve a preocupação de verificar a compreensão das participantes quanto ao conteúdo comunicado.

Nas cinco ações educativas observadas abordaram-se pílula, DIU, injetável (mensal e trimestral), esterilização cirúrgica masculina, preservativo masculino e feminino e diafragma. A esterilização cirúrgica feminina deixou de ser abordada em apenas uma ocasião, enquanto a anticoncepção de emergência nunca foi mencionada. 
Os aspectos abordados sobre cada MAC variaram nas cinco ações educativas observadas: em relação à pílula, DIU, injetável, esterilização cirúrgica feminina e masculina, em todas as ocasiões se mencionou o mecanismo de ação e a forma de uso, mas nem sempre se falou sobre contra-indicações e efeitos secundários.

\section{Discussão}

De modo geral, os resultados apresentados indicam que as mulheres sentiram-se bastante livres para escolher um MAC, consideraram que a ação educativa aumentou essa liberdade e que não lhes faltou informação sobre o método escolhido. A continuidade do uso do MAC escolhido esteve relacionada à satisfação das mulheres com o mesmo e à sua percepção quanto à suficiência das informações recebidas quando optaram por esse MAC.

Dessa forma, tendo-se em conta os elementos de Bruce ${ }^{3}$ para qualidade de atenção em planejamento familiar, os resultados de estudos recentes realizados em outros países e a realidade brasileira, pode-se considerar que as mulheres da amostra estudada receberam uma atenção de boa qualidade em regulação da fecundidade, ou planejamento familiar. A disponibilidade de uma variedade de MAC associada à qualidade da comunicação interpessoal e do aconselhamento estariam dentro dos parâmetros ideais para assegurar a liberdade de escolha, bem como proporcionar uma aproximação cliente-provedor, e conseqüentemente, maior satisfação à clientela 12,13,14 .

A qualidade da atenção em planejamento familiar tem sido reconhecida como fator essencial para o início e a continuidade do uso de MAC. Ramarao et al. 7, por exemplo, verificaram, nas Filipinas, que as mulheres que haviam recebido uma atenção de média ou alta qualidade, quando procuraram um método em serviço de planejamento familiar, apresentavam maior probabilidade de continuarem usando um MAC, em comparação com as mulheres que receberam atenção de baixa qualidade. De forma semelhante, Koening 15 observou que a qualidade de atenção era decisiva para a continuidade de uso de um contraceptivo entre mulheres da área rural de Bangladesh, especialmente entre aquelas com menor nível educacional ou sócio-econômico.

Apesar desses aspectos positivos, é preciso entender também a questão da insatisfação das mulheres estudadas como fator importante para a descontinuação do MAC. Dentre as mulheres que, após seis meses de terem inici-
Tabela 3

Distribuição percentual das entrevistadas, segundo autopercepção sobre a liberdade de escolha do MAC (métodos anticoncepcionais) que aceitaram na consulta.

\begin{tabular}{lr}
\hline Autopercepção & $\%$ \\
\hline Sentiu-se livre para escolher & 99,6 \\
Sim & 0,4 \\
Não & \\
Quão livre se sentiu* & 81,9 \\
Bastante & 16,1 \\
Mais ou menos & 2,0 \\
Pouco & \\
Influência da ação educativa e da consulta sobre a escolha* & 68,5 \\
Alta & 14,9 \\
Média & 6,9 \\
Baixa & 9,7 \\
Muito baixa & \\
Influência da ação educativa e da consulta & \\
sobre a liberdade de escolha & \\
Liberdade aumentou & 60,0 \\
Liberdade diminuiu & 0,0 \\
Não teve influência & 40,0 \\
Total & 250 \\
\hline
\end{tabular}

* Faltou informação de duas mulheres.

ado o uso de método, estavam pouco satisfeitas/insatisfeitas, metade havia descontinuado o MAC; todavia, quase três quartos consideravam que não lhes faltara informação na ação educativa e consulta. Este resultado leva a refletir sobre o que produziu a insatisfação quanto ao método escolhido. Quando se analisam as razões de insatisfação, encontram-se motivos relacionados a aspectos abordados, de fato, nas ações educativas, o que, aliás, foi reconhecido pelas mulheres, uma vez que asseguraram não ter faltado informação. Isso pode indicar que, mesmo recebendo a informação, esta não foi significativa para elas naquele momento, provavelmente porque, então, a prioridade era a eficácia do MAC (Osis MJD, Duarte GA, Bento SF. O que as mulheres pensam sobre a laqueadura tubária. Trabalho apresentado no IV Congresso Latino-Americano de Ciências Sociais e Medicina, realizado em Cocoyoc, México, em 1997). Partindo desse ponto de vista, é possível pensar que nem sempre as mulheres dão relevância a informações sobre possíveis efeitos colaterais de um contraceptivo no momento em que estão iniciando o seu uso. Porém, mais tarde, esses efeitos poderão 
Tabela 4

Distribuição percentual das entrevistadas, segundo satisfação com o MAC (métodos anticoncepcionais) iniciado e opinião sobre o que esse MAC trouxe para suas vidas e se consideravam que faltou ou não alguma informação sobre ele.

\begin{tabular}{lc}
\hline Categorias & $\%$ \\
\hline MAC em uso após 6 meses & \\
O mesmo & 87,3 \\
Nenhum/outro & 12,7 \\
& \\
Satisfação & \\
Muito satisfeita & 32,4 \\
Satisfeita & 52,6 \\
Pouco satisfeita & 7,5 \\
Insatisfeita & 7,5 \\
& \\
Faltou informação & 11,0 \\
Sim & 89,0 \\
Não & \\
O uso do MAC trouxe* & \\
Benefícios & \\
Prejuízos & \\
Um pouco de cada & \\
* Excluídas 36 mulheres que ainda estavam esperando para fazer laqueadura & \\
\hline & \\
\hline & \\
\hline
\end{tabular}

ser os principais motivos para descontinuação do uso 16 . Esse aspecto representa um verdadeiro desafio para os profissionais da saúde, no sentido de conseguirem mobilizar a atenção das mulheres para essa questão quando elas vão aos serviços solicitando um determinado MAC.

Outro aspecto que merece atenção é o fato de que praticamente todas as mulheres chegaram ao ambulatório já com um MAC escolhido, e poucas mudaram de método, apesar de a maioria afirmar que a ação educativa e a consulta influenciaram a sua escolha. Isso pode ser interpretado no sentido de que as mulheres utilizaram o conteúdo recebido na ação educativa como uma espécie de legitimador daquilo que já tinham em mente. Essa possibilidade leva a questionar o papel da ação educativa, o que é reforçado pela constatação de que só mudaram de MAC as mulheres que apresentavam algum impedimento muito significativo - legal ou de saúde - para o uso do método inicialmente pretendido. Talvez se não existissem essas condições especiais apontadas pelos profissionais de saúde, todas as mulheres teriam saído do ambulatório com o MAC desejado.

É necessário refletir, também, sobre como essas mulheres chegaram a pretender usar um determinado MAC. Os resultados deixam claro que as principais influências nesse processo de

Distribuição percentual das entrevistadas segundo a satisfação com MAC (métodos anticoncepcionais) que estavam usando após 6 meses, se consideravam que faltou ou não alguma informação sobre o MAC, e a opinião sobre o que o uso dele trouxe para suas vidas.

\begin{tabular}{|c|c|c|c|}
\hline Categorias & $\begin{array}{l}\text { Muito satisfeita/ } \\
\text { satisfeita }\end{array}$ & $\begin{array}{l}\text { Pouco satisfeita/ } \\
\text { insatisfeita }\end{array}$ & $\mathrm{p}$ \\
\hline \multicolumn{4}{|c|}{ MAC em uso após 6 meses } \\
\hline Mesmo & 94,0 & 50,0 & $<0,001$ \\
\hline Nenhum/outro & 6,1 & 50,0 & \\
\hline \multicolumn{4}{|c|}{ Faltou informação na ação educativa/consulta } \\
\hline Sim & 8,2 & 27,0 & $<0,011$ \\
\hline Não & 91,8 & 73,0 & \\
\hline O uso do MAC trouxe* & & & ** \\
\hline Benefício & 97,2 & 42,0 & \\
\hline Prejuízo & 0,7 & 29,0 & \\
\hline Um pouco de cada & 2,1 & 29,0 & \\
\hline Total ${ }^{\star \star \star}$ & 147 & 26 & \\
\hline
\end{tabular}

* Cinco mulheres referiram não saber se o uso do MAC trouxe benefícios ou não.

** Não aplicável.

*** Excluídas 76 mulheres: 36 ainda estavam esperando para fazer a laqueadura; 38 mulheres que foram descontinuadas do estudo; uma havia mudado para coito interrompido e uma mulher cujo marido havia optado pela vasectomia e a cirurgia já havia sido feita. 
decisão foram pessoas significativas, sobretudo familiares, e que o papel dos profissionais de saúde foi bastante restrito, conforme já observado em outros estudos 17,18,19. Parece que quando as mulheres chegam a um serviço de planejamento familiar trazem consigo conceitos e pré-conceitos sobre os MAC, construídos sobre informações que para elas têm maior credibilidade do que aquelas que receberão dos profissionais de saúde. Apresenta-se, portanto, a necessidade de educação em planejamento familiar como um projeto abrangente na sociedade em geral, não apenas centralizado em serviços de saúde.

\section{Resumo}

Descreve-se a percepção de 250 mulheres que solicitaram métodos anticoncepcionais (MAC) em um serviço público de saúde quanto à sua liberdade de escolha, a contribuição de uma ação educativa e de uma consulta para essa liberdade, a satisfação com o MAC escolhido e a continuidade de seu uso, depois de seis meses. Quase todas as mulheres (99,6\%) chegaram ao serviço com um MAC já escolhido e 90,0\% saíram usando esse método; $81,9 \%$ referiram ter se sentido bastante livres para escolher o MAC e 60,0\% disseram que a ação educativa e a consulta aumentaram a sua liberdade. Seis meses depois, 87,3\% continuavam usando o mesmo MAC. Entre as mulheres que declararam alguma insatisfação com o método iniciado, foi significativamente maior a proporção das que mudaram de MAC e das que disseram que faltou alguma informação quando iniciaram o uso. A ação educativa e a consulta parecem ter atuado como legitimadores de uma escolha feita antes de as mulheres procurarem o serviço, com base em informações recebidas de outras fontes. Isto, provavelmente, contribuiu para a percepção de terem tido bastante liberdade de escolha.

Métodos Anticoncepcionais; Planejamento Familiar; Serviços de Saúde

\section{Colaboradores}

M. J. D. Osis atuou em todas as etapas do desenvolvimento da pesquisa. Redigiu o texto completo do artigo, em colaboração com as demais autoras. G. A. Duarte e X. Espejo colaboraram em todas as etapas do desenvolvimento da pesquisa. Duarte colaborou principalmente na preparação das tabelas, descrição dos resultados e discussão. Espejo colaborou especificamente na discussão dos resultados. K. S. Pádua e E. R. Crespo atuaram no desenvolvimento da pesquisa, fizeram a revisão bibliográfica e contribuíram na preparação da Introdução e da Discussão.

\section{Agradecimentos}

Os autores agradecem à Organização Mundial da Saúde/Departamento de Saúde Reprodutiva e Pesquisa pelo financiamento recebido para a realização da pesquisa cujos resultados são apresentados neste trabalho. 


\section{Referências}

1. Ministério da Saúde. Planejamento familiar: manual para o gestor. Brasília: Ministério da Saúde; 2002.

2. Ministério da Saúde. Assistência em planejamento familiar: manual técnico. 4a Ed. Brasília: Secretaria de Política de Saúde, Ministério da Saúde; 2002.

3. Bruce J. Fundamental elements of the quality of care: a simple framework. New York: Population Council; 1989. (Working Papers 1).

4. Díaz J, Díaz M. Qualidade de atenção em saúde sexual e reprodutiva: estratégias para mudanças. In: Galvão L, Díaz J, organizadores. Saúde sexual e reprodutiva no Brasil: dilemas e desafios. São Paulo: Editora Hucitec/Population Council; 1999. p. 209-33.

5. Murphy E, Steele C. Client-provider interactions in family planning services: guidance from research and program experience. MAQ Paper 2000; 1:1-10.

6. Kim YM, Kols A, Mucheke S. Informed choice and decision-making in family planning counseling in Kenya. Int Fam Plan Perspect 1998; 24:4-11.

7. Ramarao S, Lacuesta M, Costello M, Pangolibay $\mathrm{B}$, Jones $\mathrm{H}$. The link between quality of care and contraceptive use. Int Fam Plan Perspect 2003; 29:76-83.

8. Costa AM. O PAISM: uma política de assistência integral à saúde da mulher a ser resgatada. São Paulo: Comissão de Cidadania e Reprodução; 1992.

9. World Health Organization. An assessment of the need for contraceptive introduction in Brazil. Geneva: World Health Organization; 1994.

10. Walsh J, Lythgoe H, Peckham S. Contraceptive choices supporting effective use of methods. London: Family Planning Association; 1995.

11. Altman DG. Practical statistics for medical research. New York: Chapman \& Hall; 1999.
12. Khan AR, Boon-Ann T, Mehta S. Quality of care and target-free approach family planning programmes for family planning programmes. http: //www.unescap.org/esid/psis/population/icpd/s ec6.asp (acessado em 22/Jul/2003).

13. Kim YM, Figueroa ME, Martin A, Silva A, Acosta SF, Hurtado M, et al. Impact of supervision and self-assessment on doctor-patient communication in rural Mexico. Int J Qual Health Care 2002; 14:359-67.

14. Kim YM, Kols A, Putjuk F, Heerey M, Rinehart W, Elwyn G, Edwards A. Participation by clients and nurse midwives in family planning decision making in Indonesia. Patient Educ Couns 2003; 50: 295-302.

15. Koening MA. The impact of quality of care on contraceptive use: evidence from longitudinal data from rural Bangladesh. http://www.dec.org/ pdf_docs/PNACU002.pdf (acessado em 10/Out/ 2003).

16. Leite IC. Descontinuação de métodos anticoncepcionais no Nordeste do Brasil, 1986-1991. Cad Saúde Pública 2003; 19:1005-16.

17. Hardy E, Goodson P, Souza TR, Rodriguez CM. Factors associated with the acceptance of Norplant or IUD among women with similar sociodemographic characteristics. Adv Contracept 1991; 7:95-105.

18. Espejo X, Tsunechiro MA, Osis MJD, Duarte GA, Bahamondes L, Sousa MH. Adequação do conhecimento sobre métodos anticoncepcionais entre mulheres de Campinas, SP. Rev Saúde Pública 2003; 37:583-90.

19. Marchi NM, Alvarenga AT, Osis MJD, Bahamondes L. Opção pela vasectomia e relações de gênero. Cad Saúde Pública 2003; 19:1017-27.

Recebido em 12/Nov/2003

Versão final reapresentada em 18/Mai/2004 Aprovado em 31/Mai/2004 